\title{
Real-Time Investigation of an Adaptive Fuzzy Synergetic Controller for a DC-DC Buck Converter
}

\author{
Khalissa Behih \\ Department of Electrical Engineering \\ Ferhat Abbas University Setif I \\ Setif, Algeria \\ khalissabehih@univ-setif.dz \\ Ziyad Bouchama \\ Department of Sciences and Technology \\ Bachir El Ibrahimi University \\ Bordj Bou Arreridj, Algeria \\ bouchama_ziad@yahoo.fr
}

\author{
Khier Benmahammed \\ LSI Laboratory \\ Ferhat Abbas University Setif I \\ Setif, Algeria \\ kierben@ieee.org \\ Mohamed Naguib Harmas \\ QUERE Laboratory \\ Ferhat Abbas University Setif I \\ Setif, Algeria \\ mharmas@yahoo.fr
}

\begin{abstract}
In this paper, an adaptive fuzzy synergetic controller (AFSC) for a DC-DC converter is introduced. Two robust control techniques, synergetic control combined with fuzzy logic control, are utilized to produce a reliable DC-DC power supply. This isrealized by an indirect adaptive control of a DC-DC buck converter. To further enhance robustness, a nonlinear constraint, the equivalent of the sliding surface, is used to guarantee performance and stability for any operating condition. To ensure overall strength, closed-loop signals are bounded and the stability is guaranteed using the Lyapunov theory. Control parameters are optimized using a PSO algorithm to further enhance performances. The proposed controller (AFSC) is designed through a dSpace based experimental setup to provide robust DC-DC buck converter voltage control.
\end{abstract}

Keywords-adaptive; fuzzy control; synergetic control; DC-DC converter; PSO algorithm; dSpaceboard DS1104

\section{INTRODUCTION}

Reliable power supply is indispensable in any energetic system and computing unit. DC-DC converters represent a dependable power source used in many computer-based industrial plants as well as in renewable energy systems control [1-3]. Failure or chaotic behavior may occur in these subsystems, often resulting in catastrophic repercussions [4-5]. Therefore DC-DC converter robust operation is essential for a wide operating range and for different loading conditions [6-7]. Parameter variations and varying operating points require a robust modeling approach. Fuzzy systems have been proven to adequately handle systems with partially known or unknown system models using the universal approximation theorem [811] and will be exploited to approximate nonlinear system dynamics. Added robustness in controlling such systems has been achieved using sliding mode control despite unwanted chattering. Many sound approaches have been elaborated to alleviate [12-13] this unsafe drawback or to eliminate it completely, often at the expense of robustness. Synergetic control, providing a robust and continuous control law, has emerged recently [14-16] as a promising technique. It relies on the same principles with sliding mode control and provides similar robustness but without discontinuous components in its control signal, therefore eliminating chattering.

Buck converter is the name given to a step-down DC-DC converter producing a lower average voltage at the output than the input supply voltage. Its control performance must be upheld despite load and parameter variations occurring in many applications starting from basic computers to space vehicles or nuclear submarines. Many control methods have been used, such as PID [17], fuzzy control [18], feedback linearization [19], and many others schemes using a precise model and fixed control parameters which can be detrimental to system performance [20]. The principal contribution of this paper consists in combining fuzzy inference systems and synergetic control to provide a reliable and robust DC-DC converter voltage control despite operating point excursions and parameter uncertainties. The adaptive scheme ensures robustness despite system parameter fluctuations while synergetic approach permits imposing system designer chosen dynamics without incurring devastating chattering inherent to sliding mode methodology. Controller parameters are obtained with the use of particle swarm optimization (PSO) algorithm and closed-loop stability is insured utilizing Lyapunov synthesis. Results are given for DC-DC converter control for different operating conditions showing remarkable performance.

\section{MODEL OF THE DC-DC CONVERTER}

The basic buck DC-DC converter topology is shown in Figure 1. When the converter works in conduction mode, the system can be described by: 


$$
\left\{\begin{aligned}
\dot{i}_{L}(t)= & -\frac{1}{L}\left(r_{L}+\frac{R r_{C}}{R+r_{C}}\right) i_{L}(t)-\frac{R}{L\left(R+r_{C}\right)} u_{c}(t) \\
& -\frac{v_{D}}{L}+\frac{1}{L}\left(v_{D}+V_{i n}+r_{m} i_{L}(t)\right) d \\
\dot{u}_{c}(t)= & \frac{R}{C\left(R+r_{C}\right)} i_{L}(t)+\frac{1}{C\left(R+r_{C}\right)} u_{c}(t)
\end{aligned}\right.
$$

where $i_{L}$ is the average inductor current, $u_{c}$ is the average capacitor voltage and $d$ is the control signal. The positive constants $R, L, C$ and $V_{i n}$ are the load resistance, inductance, capacitance and input voltage source while $r_{L}, r_{C}, r_{m}$, and $v_{D}$ are parasitic elements.

The output voltage $v_{0}(t)$ can be determined from:

$$
v_{0}(t)=\frac{R r_{C}}{R+r_{C}} i_{L}(t)+\frac{R}{\left(R+r_{C}\right)} u_{c}(t)
$$

The control law objective is to drive the circuit with a desired constant output voltage regardless of load and line input variations. Tracking error and its derivative are defined as follows:

$$
\left\{\begin{array}{l}
e(t)=v_{0}-v_{r e f} \\
\dot{e}(t)=f\left(i_{L}, u_{c}\right)+g\left(i_{L}, u_{c}\right) d-\dot{v}_{r e f}
\end{array}\right.
$$

where $v_{r e f}$ is the desired output voltage, $f\left(i_{L}, u_{c}\right)$ and $g\left(i_{L}, u_{c}\right)$ are variable functions of state system and circuit components [21]. The adaptive fuzzy synergetic control applied to a buck DC-DC converter is developed in the next section.

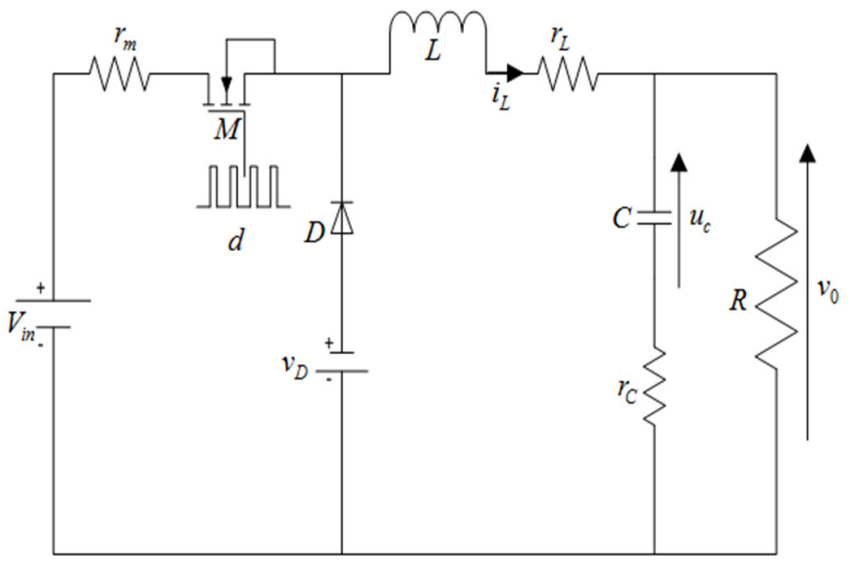

Fig. 1. Buck DC-DC converter schematics

\section{DC-DC CONVERTER FUZZY SYNERGETIC CONTROL}

Synergetic control synthesis of the system given in (1) begins by defining a designer chosen macro-variable given as in (4):

$$
\Psi=e+\beta \int e d t
$$

where $\beta$ is a positive constant.

Let the desired dynamic evolution of the macro-variable be designer imposed as:

$$
\alpha \dot{\Psi}+\Psi=0
$$

where $\alpha$ is a positive constant. Taking the first time derivative on the macro-variable function leads to:

$$
\dot{\Psi}=\dot{e}+\beta e
$$

which can be rewritten as:

$$
\dot{\Psi}=f\left(i_{L}, u_{c}\right)+g\left(i_{L}, u_{c}\right) d+\beta e-\dot{v}_{r e f}
$$

Combining (6) and (8) yields:

$$
f\left(i_{L}, u_{c}\right)+g\left(i_{L}, u_{c}\right) d+\beta e-\dot{v}_{r e f}+=-\frac{1}{\alpha} \Psi
$$

Solving for the synergetic control law leads to:

$$
d=-\left(\frac{1}{\alpha} \Psi+\beta e-\dot{v}_{r e f}+f\left(i_{L}, u_{c}\right)\right) g\left(i_{L}, u_{c}\right)^{-1}
$$

The control law in (10) assumes that $f\left(i_{L}, u_{c}\right)$ and $g\left(i_{L}, u_{c}\right)$ are perfectly known and that the system is operating in the controllability region, that is $g\left(i_{L}, u_{c}\right)^{-1} \neq 0$. In the more realistic case where $f\left(i_{L}, u_{c}\right)$ and $g\left(i_{L}, u_{c}\right)$ are unknown, the control law (10) for DC-DC buck converter must be modified as:

$$
d_{F}=-\left[\frac{1}{\alpha} \Psi+\beta e-\dot{v}_{r e f}+\hat{f}\left(x / \theta_{f}\right)\right] \hat{g}\left(x / \theta_{g}\right)^{-1}
$$

where $x=\left[\begin{array}{ll}i_{L} & u_{c}\end{array}\right]^{T}$ is a vector of measurable states and the fuzzy estimates function $\hat{f}\left(x / \theta_{f}\right)$ and $\hat{g}\left(x / \theta_{g}\right)$ are defined as [8-9]:

$$
\begin{aligned}
& \hat{f}\left(x / \theta_{f}\right)=\theta_{f}^{T} \xi(x) \\
& \hat{g}\left(x / \theta_{g}\right)=\theta_{g}^{T} \xi(x)
\end{aligned}
$$

where $\xi(x)$ is the fuzzy basis function defined as:

$$
\xi_{l}(x)=\frac{\prod_{i=1}^{n} \mu_{F_{i}^{l}}\left(x_{i}\right)}{\sum_{l=1}^{M}\left(\prod_{i=1}^{n} \mu_{F_{i}^{l}}\left(x_{i}\right)\right)}
$$

$\mu_{F_{i}^{l}}\left(x_{i}\right)$ represents the membership function value of $x_{i}$ and 
$F_{i}^{l}$ are labels of fuzzy sets in $U=\prod_{i=1}^{n} U_{i} \in R^{n}$. Fuzzy logic systems' vectors $\theta_{f}$ and $\theta_{g}$ can be continuously updated as in $[13,22-23]$ :

$$
\begin{aligned}
& \dot{\theta}_{f}=\kappa_{f} \Psi \xi(x) \\
& \dot{\theta}_{g}=\kappa_{g} \Psi \xi(x) d
\end{aligned}
$$

where $\kappa_{f}$ and $\kappa_{g}$ represent adaptive positive learning rates. Fuzzy system sets can provide up to the desired accuracy approximation as detailed in $[8-9,13,22]$.

\section{STABILITY AND ROBUSTNESS ANALYSIS}

Using the control action (11) in system (1), with $\hat{f}$ and $\hat{g}$ defined in (12) and (13), while parameters vectors $\theta_{f}$ and $\theta_{g}$ are adjusted by the adaptive laws (15) and (16) the closed loop system signals are bounded and the tracking voltage error converge asymptotically to zero.

Proof: The stability and robustness issue of the controller are addressed here by using Lyapunov stability theory as follows:

First, defining the optimal parameters of the fuzzy system:

$$
\begin{gathered}
\hat{\theta}_{f}=\arg \min _{\theta_{f} \in \Omega_{f}}\left[\sup _{x \in R^{n}}\left|\hat{f}\left(x / \theta_{f}\right)-f(x)\right|\right] \\
\hat{\theta}_{g}=\arg \min _{\theta_{g} \in \Omega_{g}}\left[\sup _{x \in R^{n}}\left|\hat{g}\left(x / \theta_{g}\right)-g(x)\right|\right]
\end{gathered}
$$

where $\Omega_{f}$ and $\Omega_{g}$ are constraint sets for $\theta_{f}$ and $\theta_{g}$, respectively, then (8) can be rewritten as:

$$
\begin{gathered}
\dot{\Psi}(t)=\beta e+f(x)+\hat{g}\left(x / \hat{\theta}_{g}\right) d_{F}+g(x) d-\hat{g}\left(x / \hat{\theta}_{g}\right) d_{F} \\
=\varepsilon+\left(\hat{\theta}_{f}^{T}-\theta_{f}^{T}\right) \xi(x)+\left(\hat{\theta}_{g}^{T}-\theta_{g}^{T}\right) \xi(x) d_{F}-\frac{1}{\alpha} \Psi
\end{gathered}
$$

where $\dot{v}_{r e f}=0$, and $\varepsilon$ is the minimum approximation error defined by:

$$
\varepsilon=f(x)-\hat{f}\left(x / \hat{\theta}_{f}\right)+\left(g(x)-\hat{g}\left(x / \hat{\theta}_{f}\right)\right) d_{F}
$$

Letting $\varphi_{f}=\left(\hat{\theta}_{f}-\theta_{f}\right)$ and $\varphi_{g}=\left(\hat{\theta}_{g}-\theta_{g}\right)$ one may rewrite (19) as:

$$
\dot{\Psi}=\varepsilon+\varphi_{f}^{T} \xi(x)+\varphi_{g}^{T} \xi(x) \xi(x) d_{F}-\frac{1}{\alpha} \Psi
$$

Defining Lyapunov function candidate:

$$
V=\frac{1}{2}\left(\Psi^{2}+\frac{1}{\kappa_{f}} \varphi_{f}^{T} \varphi_{f} \frac{1}{\kappa_{g}} \varphi_{g}^{T} \varphi_{g}\right)
$$

the time derivative of $V$ leads to:

$$
\begin{aligned}
\dot{V}= & \Psi \dot{\Psi}+\frac{1}{\kappa_{f}} \varphi_{f}^{T} \dot{\varphi}_{f}+\frac{1}{\kappa_{g}} \varphi_{g}^{T} \dot{\varphi}_{g} \\
= & \Psi\left(\varepsilon+\varphi_{f}^{T} \xi(x)+\varphi_{g}^{T} \xi(x) d_{F}-\frac{1}{\alpha} \Psi\right) \\
& +\frac{1}{\kappa_{f}} \varphi_{f}^{T} \dot{\varphi}_{f}+\frac{1}{\kappa_{g}} \varphi_{g}^{T} \dot{\varphi}_{g} \\
\leq & -\frac{1}{\alpha} \Psi^{2}+\frac{1}{\kappa_{f}} \varphi_{f}^{T}\left(\kappa_{f} \Psi \xi(x)+\dot{\varphi}_{f}\right) \\
& +\frac{1}{\kappa_{g}} \varphi_{g}^{T}\left(\kappa_{g} \Psi \xi(x) d_{F}+\dot{\varphi}_{g}\right)+\Psi \varepsilon
\end{aligned}
$$

where $\dot{\varphi}_{f}=-\dot{\theta}_{f}$ and $\dot{\varphi}_{g}=-\dot{\theta}_{g}$. Substituting (15) and (16) in (23), leads to:

$$
\dot{V} \leq-\frac{1}{\alpha} \Psi^{2}+\Psi \varepsilon
$$

Based on the universal approximation theorem [8-9], if $\varepsilon \in L_{2}$, then $\Psi \in L_{2}$. From (17) and (18), one concludes that $\Psi$ is bounded as well as every term in (24). Hence, $\Psi, \dot{\Psi} \in L_{\infty}$, and using Barbalat lemma [24-25] leads to: $\lim _{t \rightarrow \infty}|\Psi|=0$ while the term $\Psi \varepsilon$, in (24) is very small such that $\dot{V} \leq 0$. The error will asymptotically converge to zero. The stability of system (1) is therefore guaranteed.

The control parameters used were selected on a trial-error basis thus not leading to optimum performance. This issue is addressed in the ensuing section using the PSO algorithm.

\section{PARTICLE SWARM OPTIMIZATION}

Traditional optimization techniques among which linear programming, nonlinear programming, quadratic programming, and interior point methods are very sensitive to starting points and often diverge or converge to local optimums. More global nature-inspired optimization approaches have been developed [26-27]. PSO, as evolutionary algorithm and genetic algorithm, is a heuristic and global search method which explores the search space to get to the global optimum, using a population-based algorithm modelled on swarm intelligence.

Seeking further enhancement of system control performance, optimization techniques have been used successfully in buck DC-DC converter [28]. In the present work, PSO [29-32] is used to minimize an objective function thus obtaining optimum parameter settings of the proposed DC-DC converter fuzzy synergetic controller. The selected objective function to be minimized (ITAE) is defined by:

$$
\text { ITAE }=\int_{0}^{t} t\left|v_{0}-v_{r e f}\right| d t
$$

where $t$ is the time range of simulation and $\left|v_{0}-v_{\text {ref }}\right|$ the absolute value of the voltage error. 
A simplified schematic diagram of the proposed controller AFSC and the interconnection of the techniques used is illustrated in Figure 2. Control parameters to be tuned through the optimization algorithm are $a$ and $\beta$, with the aim to minimize the selected fitness objective function improving system response performance in terms of voltage tracking error. The objective function (25) evolution is shown in Figure 3.

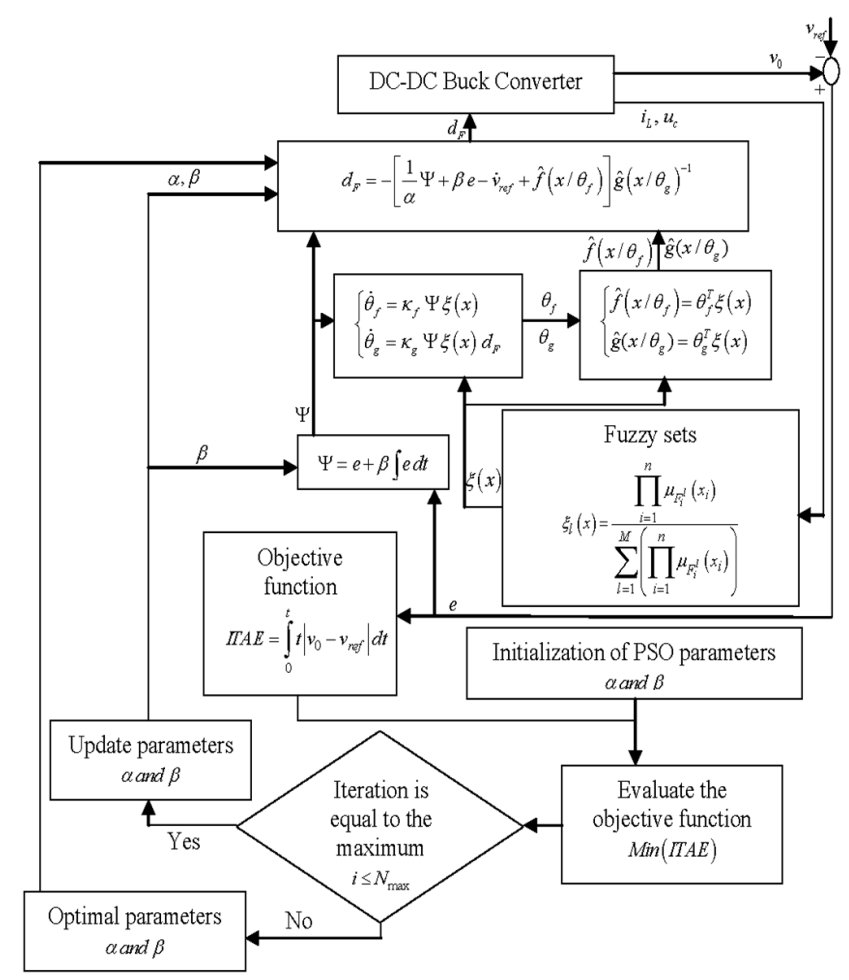

Fig. 2. Overall scheme of the optimal AFSC controller for a DC-DC buck converter

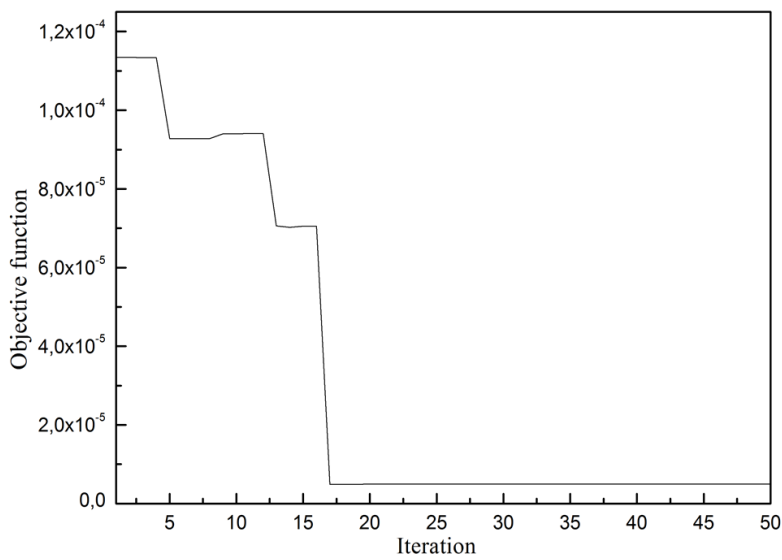

Fig. 3. Objective function evolution

\section{Simulation RESUlts}

Simulation results are given for a DC-DC converter with given parameters: $V_{i n}=30 \mathrm{~V}, L=50 \mathrm{mH}, C=470 \mu \mathrm{F}, R=25 \Omega$ and $v_{r e f}=12 \mathrm{~V}$. Optimized values for $a$ and $\beta$ are sought through PSO and they were found to be: $a=0.3210$ and $\beta=64.01$ as shown in Figures 4-5. Performance and robustness of the proposed AFSC for the DC-DC buck converter are investigated experimentally for different operating conditions through the use of a dSpace board DS1104 and a DC-DC buck converter. The experimental results are shown in Figures 6-8 with uncertainties on the system parameters.

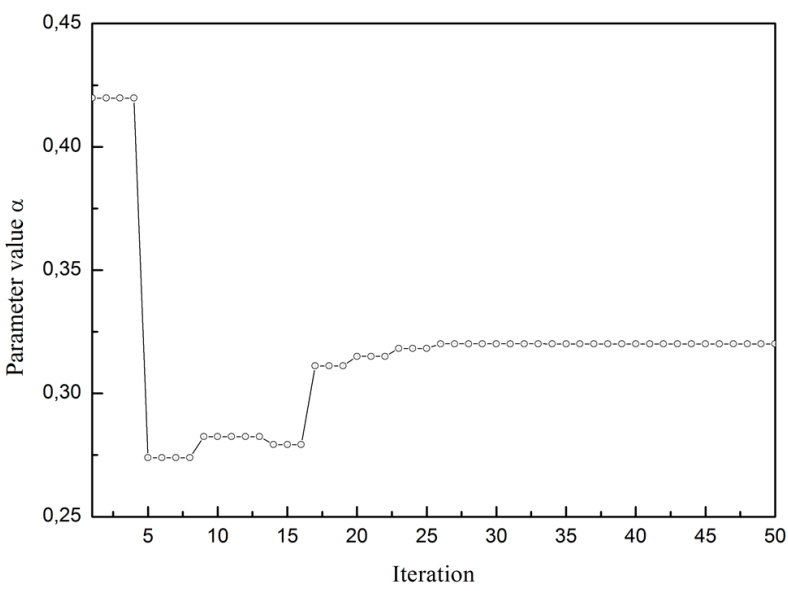

Fig. 4. The parameter value trajectory $\alpha$

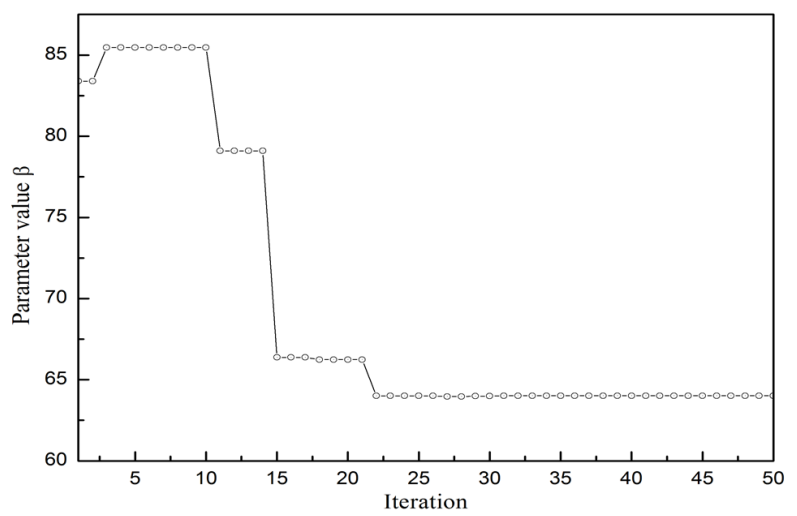

Fig. 5. The parameter value trajectory $\beta$

A load disturbance was considered in the first case, in which the system is subjected to strong perturbation as the load changes abruptly and periodically, the output voltage remains insensitive to fluctuations while inductor current follows its desired value with no steady-state error as shown in Figure 6. Considering the reference output voltage changes, from $v_{\text {ref }}=12 \mathrm{~V}$ to $v_{\text {ref }}=5 \mathrm{~V}$ the corresponding results are shown in Figure 7 . It can be easily concluded that the proposed controller achieves strong robustness and has satisfactory response under these types of disturbance and uncertainties. Finally, further experimental test is carried out for a severe operating condition such as line variation from $v_{i n}=45 \mathrm{~V}$ to $v_{i n}=24 \mathrm{~V}$. The system responses are shown in Figure 8. From the results, one can see that AFSC has remarkable performance despite the introduced perturbations. 


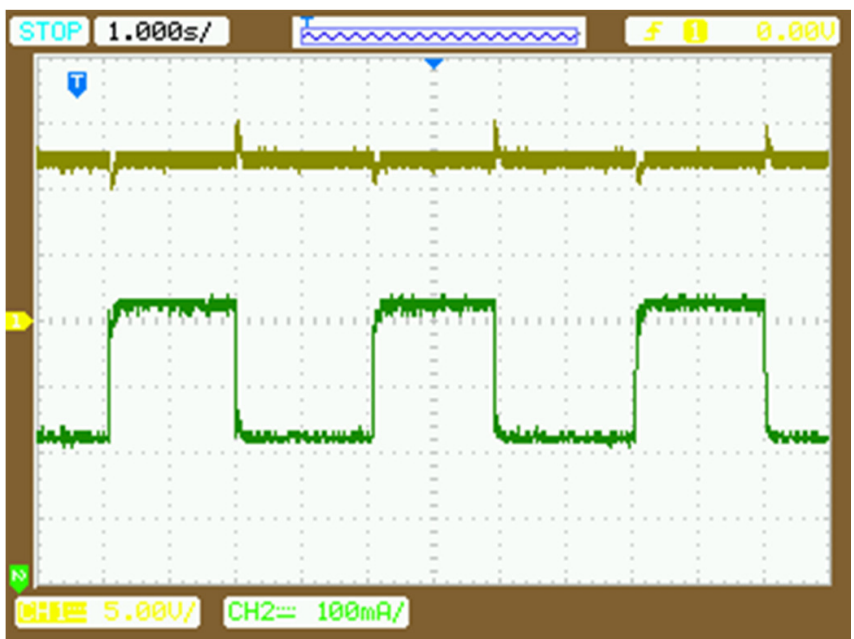

Fig. 6. Inductor current response (green line) and output voltage response (dark yellow line)

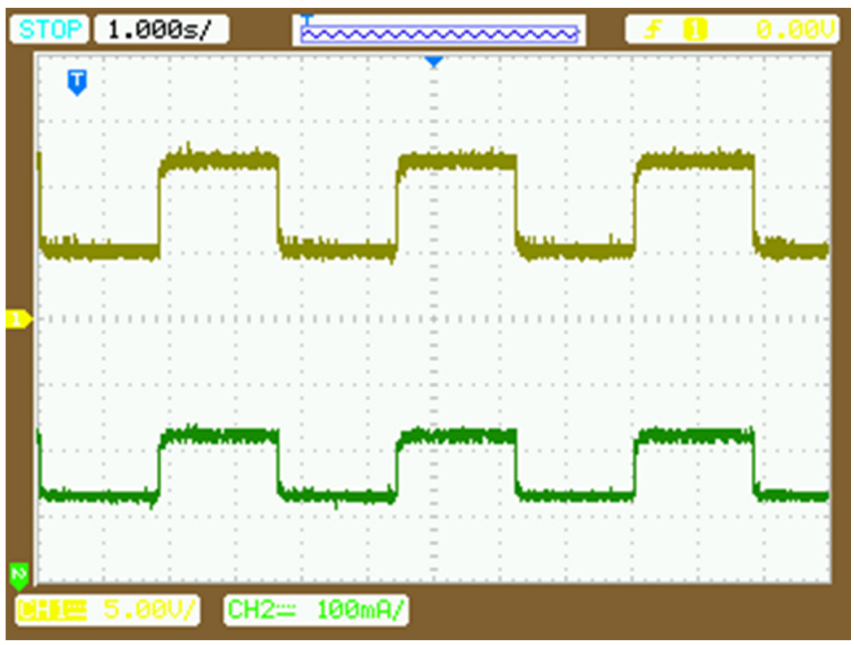

Fig. 7. Inductor current response (green line) and output voltage response (dark yellow line)

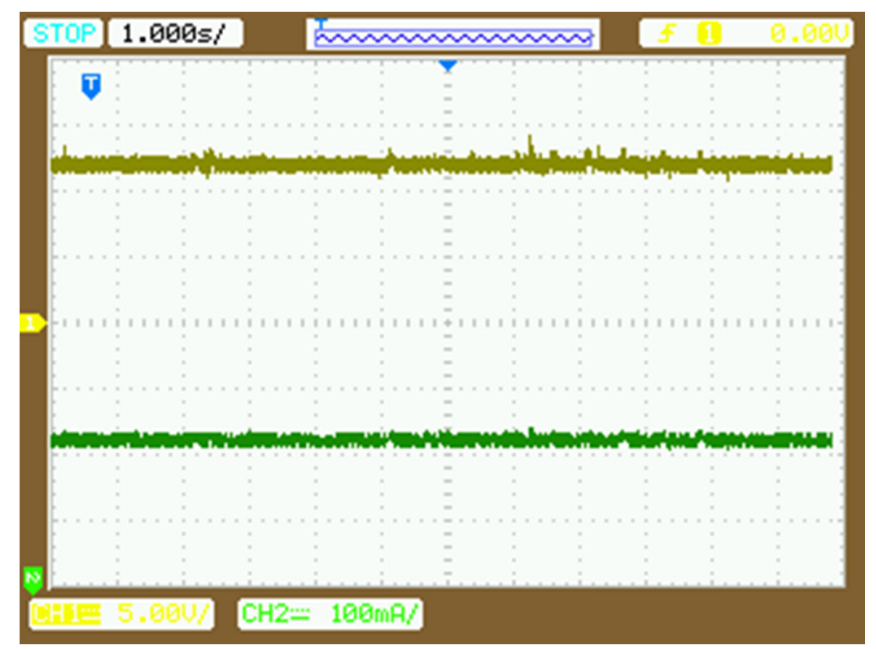

Fig. 8. Inductor current response (green line) and output voltage response (dark yellow line)

\section{CONCLUSION}

An adaptive fuzzy synergetic control scheme for a DC-DC buck converter has been presented. Its stability has been provided through Lyapunov synthesis with controller parameters optimized by PSO algorithm. The real time implementation of the proposed approach overcame the necessity of a fully known mathematical model of the DC-DC converter even though the latter has been subjected to different operating conditions showing good overall performance, improved tracking, and faster response.

\section{REFERENCES}

[1] S. Mohamadian, M. H. Khanzade, "A five-level current-source inverter for grid-connected or high-power three-phase wound-field synchronous motor drives", Engineering, Technology \& Applied Science Research, Vol. 6, No. 5, pp. 1139-1148, 2016

[2] A. Abdelrehim, S. D. Erfan, M. E. Habrouk, K. H. Youssef, "Indirect 3d-space vector modulation for matrix converter", Engineering, Technology \& Applied Science Research, Vol. 8, No. 2, pp. 2847-2852, 2018

[3] Z. R. Labidi, H. Schulte, A. Mami, "A model-based approach of dc-dc converters dedicated to controller design applications for photovoltaic generators", Engineering, Technology \& Applied Science Research, Vol. 9, No. 4, pp. 4371-4376, 2019

[4] G. Poddar, K. Chakrabarty, S. Banerjee, "Control of chaos in dc-dc converters", IEEE Transactions on Circuits and Systems I: Fundamental Theory and Applications, Vol. 45, No. 6, pp. 672-676, 1998

[5] X. Zhou, J. Li, Y. Ma, "Chaos phenomena in dc-dc converter and chaos control”, Procedia Engineering, Vol. 29, pp. 470-473, 2012

[6] S. C. Tan, Y. M. Lai, C. K. Tse, Sliding mode control of switching power converters: Techniques and implementation, CRC Press, 2017

[7] S. M. M. Shariatmadar, S. M. J. Jafarian, "Application of minimum-time optimal control system in buck-boost bi-linear converters", Engineering, Technology \& Applied Science Research, Vol. 7, No. 4, pp. 1753-1758, 2017

[8] L. X. Wang, "Stable adaptive fuzzy control of nonlinear systems", IEEE Transactions on Fuzzy Systems, Vol. 1, No. 2, pp. 146-155, 1993

[9] L. X. Wang, "Stable adaptive fuzzy controllers with application to inverted pendulum tracking", IEEE Transactions on Systems, Man, and Cybernetics, Part B (Cybernetics), Vol. 26, No. 5, pp. 677-691, 1996

[10] B. Chen, X. P. Liu, S. S. Ge, C. Lin, "Adaptive fuzzy control of a class of nonlinear systems by fuzzy approximation approach", IEEE Transactions on Fuzzy Systems, Vol. 20, No. 6, pp. 1012-1021, 2012

[11] X. Zhao, X. Wang, G. Zong, H. Li, "Fuzzy-approximation-based adaptive output-feedback control for uncertain nonsmooth nonlinear systems", IEEE Transactions on Fuzzy Systems, Vol. 26, No. 6, pp. 3847-3859, 2018

[12] A. A. Khazraji, N. Essounbouli, A. Hamzaoui, F. Nollet, J. Zaytoon, "Type-2 fuzzy sliding mode control without reaching phase for nonlinear system", Engineering Applications of Artificial Intelligence, Vol. 24, No. 1, pp. 23-38, 2011

[13] Z. Bouchama, N. Essounbouli, M. N. Harmas, A. Hamzaoui, K. Saoudi, "Reaching phase free adaptive fuzzy synergetic power system stabilizer", International Journal of Electrical Power \& Energy Systems, Vol. 77, pp. 43-49, 2016

[14] E. Santi, A. Monti, D. Li, K. Proddutur, R. A. Dougal, "Synergetic control for dc-dc boost converter: Implementation options", IEEE Transactions on Industry Applications, Vol. 39, No. 6, pp. 1803-1813, 2003

[15] J. W. Shim, Y. Cho, S. J. Kim, S. W. Min, K. Hur, "Synergistic control of SMES and battery energy storage for enabling dispatchability of renew able energy sources", IEEE Transactions on Applied Superconductivity, Vol. 23, No. 3, Article ID 5701205, 2013 
[16] Z. Bouchama, M. N. Harmas, K. Zehar, "Finite time nonlinear control for dc-dc converters", Soft Computing \& Electrical Engineering, Vol. 1, No. 1, pp. 36-45, 2019

[17] L. Guo, J. Y. Hung, R. M. Nelms, "Evaluation of Dsp-based PID and fuzzy controllers for Dc-Dc converters", IEEE Transactions on Industrial Electronics, Vol. 56, No. 6, pp. 2237-2248, 2009

[18] Z. B. Duranay, H. Guldemir, S. Tuncer, "Fuzzy sliding mode control of Dc-Dc boost converter", Engineering, Technology \& Applied Science Research, Vol. 8, No. 3, pp. 3054-3059, 2018

[19] Z. R. Labidi, H. Schulte, A. Mami, “A systematic controller design for a photovoltaic generator with boost converter using integral state feedback control”, Engineering, Technology \& Applied Science Research, Vol. 9, No. 2, pp. 4030-4036, 2019

[20] N. Zerroug, M. N. Harmas, S. Benaggoune, Z. Bouchama, K. Zehar, "DSP-based implementation of fast terminal synergetic control for a DcDc buck converter", Journal of the Franklin Institute, Vol. 355, No. 5, pp. 2329-2343, 2018

[21] C. S. Chiu, C. T. Shen, "Finite-time control of Dc-Dc buck converters via integral terminal sliding modes", International Journal of Electronics, Vol. 99, No. 5, pp. 643-655, 2012

[22] Z. Bouchama, M. N. Harmas, "Optimal robust adaptive fuzzy synergetic power system stabilizer design”, Electric Power Systems Research, Vol. 83 , No. 1, pp. 170-175, 2012

[23] K. Behih, Z. Bouchama, K. Saoudi, "Finite-time fuzzy synergetic power system regulator", Soft Computing and Electrical Engineering, Vol. 1, No. 1, pp. 69-78, 2019

[24] M. Sun, "A Barbalat-like lemma with its application to learning control", IEEE Transactions on Automatic Control, Vol. 54, No. 9, pp. 2222-2225, 2009

[25] M. Hou, G. Duan, M. Guo, "New versions of Barbalats lemma with applications", Journal of Control Theory and Applications, Vol. 8, No. 4, pp. $545-547,2010$

[26] S. Murawwat, I. Manzoor, H. Akash, J. Ahmed, "Energy cycle optimization of mobile phones using a sleep cycle optimization algorithm", Engineering, Technology \& Applied Science Research, Vol. 9, No. 4, pp. 4389-4391, 2019

[27] I. Marouani, A. Boudjemline, T. Guesmi, H. H. Abdallah, “A modified artificial bee colony for the non-smooth dynamic economic/environmental dispatch", Engineering, Technology \& Applied Science Research, Vol. 8, No. 5, pp. 3321-3328, 2018

[28] C. H. Cheng, P. J. Cheng, M. J. Xie, "Current sharing of paralleled DcDc converters using GA-based PID controllers", Expert Systems with Applications, Vol. 37, No. 1, pp. 733-740, 2010

[29] Y. Shi, R. C. Eberhart, "Empirical study of particle swarm optimization", Congress on Evolutionary Computation-CEC99 (Cat. No. 99TH8406), Washington, USA, July 6-9, 1999

[30] J. Kennedy, Particle swarm optimization, Encyclopedia of Machine Learning, Springer, 2010

[31] K. Teeparthi, D. M. V. Kumar, "Dynamic power system security analysis using a hybrid PSO-APO algorithm”, Engineering, Technology \& Applied Science Research, Vol. 7, No. 6, pp. 2124-2131, 2017

[32] D. N. Truong, V. T. Bui, "Hybrid PSO-optimized ANFIS-based model to improve dynamic voltage stability", Engineering, Technology \& Applied Science Research, Vol. 9, No. 4, pp. 4384-488, 2019 